\title{
MODELING AND IDENTIFICATION OF NONLINEAR CASCADE AND SANDWICH SYSTEMS WITH GENERAL BACKLASH
}

\author{
Jozef Vörös ${ }^{*}$
}

\begin{abstract}
The paper deals with modeling and identification of nonlinear cascade and sandwich systems including general backlash, where instead of the straight lines determining the upward and downward parts of backlash characteristic, general curves are considered. This enables more precise modeling of mechanical parts and improves the performance of control systems. The analytical description of the general backlash leads to mathematical models of the cascade system with output general backlash and the sandwich system with internal general backlash, where all the model parameters are separated. Hence, the identification is solved as a quasi-linear problem. Iterative algorithms with internal variables estimation are proposed and illustrative examples are included.
\end{abstract}

K e y w or d s: nonlinear systems, general backlash, modeling, identification, cascade systems

\section{INTRODUCTION}

Control systems performance in many cases is limited by the so-called backlash. The backlash is a dynamic nonlinearity and appears mainly in gear transmissions and similar mechanical components. This kind of hard (ie non-differentiable) nonlinearity may often cause delays, oscillations and inaccuracy and the compensation of backlash has attracted research effort of several decades $[1,2]$. As the knowledge of backlash characteristic is fundamental for its compensation, the identification of systems with backlash is of key importance. Unfortunately, in many applications the backlash parameters are either poorly known or completely unknown.

There are some contributions in the literature on the identification of systems with backlash, however it is assumed that the backlash is "straight" ie, straight lines approximate the upward and downward curves of the characteristic; see eg [3-9]. This simplifies the system description, however, in some cases it leads to inaccuracies.

To increase the accuracy of control system descriptions, it may be appropriate to generalize the backlash and consider general upward and downward curves instead of straight lines. For example, backlash in gears and other mechanical components can change with time and wear; hence, the use of "general backlash" will lead to more precise system descriptions. Only few works are dealing with the identification of cascade systems with general input backlash nonlinearities [10-13]. However, no paper was published dealing with the identification of cascade systems with output general backlash in timedomain, and no paper was published dealing with the identification of sandwich systems with this type of nonlinearity.

In this paper, the identification of cascade systems with output general backlash and sandwich systems with internal general backlash based on new mathematical models are described. The proposed iterative algorithms enable simultaneous estimation of the general backlash parameters and the parameters of the included linear dynamic systems on the basis of available input and output data.

For modeling of the general backlash, a recently proposed analytic description of this dynamic nonlinearity is used, which is based on appropriate switching functions and their complements [12]. The identification method for cascade systems consisting of a linear dynamic system followed by a general output backlash is based on a special system description, where the parameters of linear dynamic system and the parameters characterizing the general backlash are separated; hence, the estimation of parameters is solved as a quasi-linear problem adopting an iterative algorithm with internal variable estimation. The identification of sandwich systems where the general backlash is preceded with a linear dynamic system and also followed by another linear dynamic system is a direct extension of the above case and the sandwich system parameters can be estimated iteratively based on available inputs and outputs. Simulation studies of cascade systems with general output backlash and sandwich systems with general internal backlash identifications are included to demonstrate the feasibility of proposed methods.

\section{GENERAL BACKLASH MODEL}

In the case of "straight" backlash, the left and right branches of the characteristic are considered to be straight lines. However, in some applications the straight lines are only advantageous approximations of general curves constituting the left and right branches of backlash as shown in Fig. 1.

\footnotetext{
* Slovak Technical University, Faculty of Electrical Engineering and Information Technology, Institute of Robotics and Cybernetics, Ilkovičova 3, 81219 Bratislava, Slovakia. jvoros@elf.stuba.sk, jvoroself@gmail.com
} 


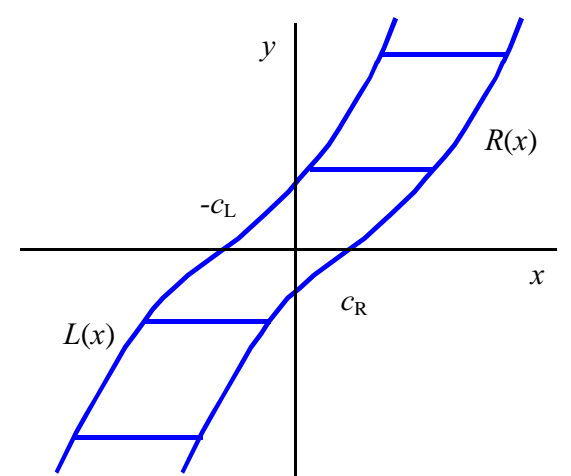

Fig. 1. General backlash characteristic

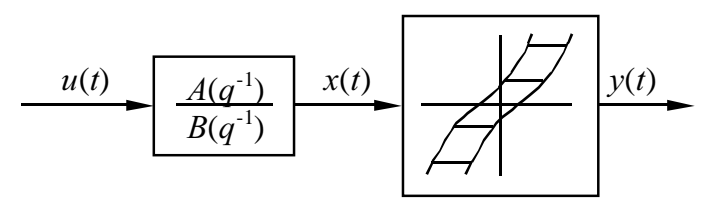

Fig. 2. Cascade system with general output backlash

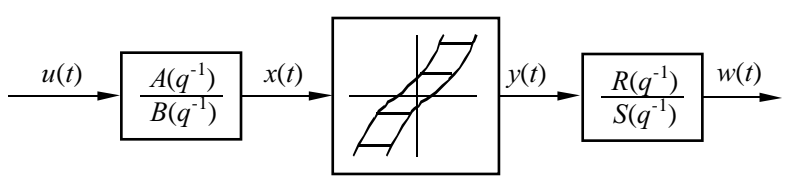

Fig. 3. Sandwich system with general backlash

The general backlash characteristic can be described by the equation $[12]$

$$
y(t)= \begin{cases}L[x(t)], & x(t) \leq z_{L} \\ y(t-1), & z_{L} \leq x(t) \leq z_{R} \\ R[x(t)], & x(t) \geq z_{R}\end{cases}
$$

where the mappings $L[x(t)]$ and $R[x(t)]$ describe the left and right branches of the characteristic, respectively, the $x$-axis values $z_{L}$ and $z_{R}$ are given as

$$
\begin{aligned}
& y(t-1)=L\left(z_{L}\right), \\
& y(t-1)=R\left(z_{R}\right) .
\end{aligned}
$$

Assume the left and right curves can be described by the polynomials

$$
\begin{aligned}
& L[x(t)]=\sum_{i=1}^{n} m_{L i}\left[x(t)+c_{L}\right]^{i}, \\
& R[x(t)]=\sum_{i=1}^{n} m_{R i}\left[x(t)-c_{R}\right]^{i},
\end{aligned}
$$

respectively, where $c_{L}>0, c_{R}>0$ are the intersections of $L[x(t)]$ and $R[x(t)]$ with the $x$-axis. Then the general backlash characteristic can be written as

$$
y(t)= \begin{cases}\sum_{i=1}^{n} m_{L i}\left[x(t)+c_{L}\right]^{i}, & x(t) \leq z_{L}, \\ y(t-1), & z_{L} \leq x(t) \leq z_{R}, \\ \sum_{i=1}^{n} m_{R i}\left[x(t)-c_{R}\right]^{i}, & x(t) \geq z_{R}\end{cases}
$$

where

$$
\begin{aligned}
& y(t-1)=\sum_{i=1}^{n} m_{L i}\left[z_{L}+c_{L}\right]^{i}, \\
& y(t-1)=\sum_{i=1}^{n} m_{R i}\left[z_{R}-c_{R}\right]^{i} .
\end{aligned}
$$

After introducing the internal variables

$$
\begin{aligned}
& \xi_{1}(t)=x(t)+c_{L}, \\
& \xi_{2}(t)=x(t)-c_{R}
\end{aligned}
$$

and considering the function

$$
h(s)= \begin{cases}0, & \text { if } s>0, \\ 1, & \text { if } s \leq 0,\end{cases}
$$

switching between two sets of values, ie, $(-\infty, s)$ and $(s, \infty)$, the following variables based on (7) and (8) can be defined

$$
\begin{aligned}
& f_{1}(t)=h\left[\sum_{i=1}^{n} m_{L i} \xi_{1}^{i}(t)-y(t-1)\right], \\
& f_{2}(t)=h\left[y(t-1)-\sum_{i=1}^{n} m_{R i} \xi_{2}^{i}(t)\right] .
\end{aligned}
$$

Then the general backlash can be characterized by one difference equation

$$
\begin{aligned}
& y(t)=\sum_{i=1}^{n} m_{L i} \xi_{1}^{i}(t) f_{1}(t)+\sum_{i=1}^{n} m_{R i} \xi_{2}^{i}(t) f_{2}(t)+ \\
& y(t-1)\left[1-f_{1}(t)\right]\left[1-f_{2}(t)\right] .
\end{aligned}
$$

To include the deadzone parameters $c_{L}$ and $c_{R}$ into the backlash model, we separate the first terms of the sums in (14) and then half-substitute from (9) and (10) as follows

$$
\begin{array}{r}
y(t)=m_{L 1} x(t) f_{1}(t)+m_{L 1} c_{L} f_{1}(t)+\sum_{i=2}^{n} m_{L i} \xi_{1}^{i}(t) f_{1}(t)+ \\
m_{R 1} x(t) f_{2}(t)-m_{R 1} c_{R} f_{2}(t)+\sum_{i=2}^{n} m_{R i} \xi_{2}^{i}(t) f_{2}(t)+ \\
y(t-1)\left[1-f_{1}(t)\right]\left[1-f_{2}(t)\right] .
\end{array}
$$

Now the input/output relation for the general backlash (15) is identical with that of (1). All the backlash parameters are separated and the equation is linear in the input, output and internal variables. This description allows the upward and downward curves to be different provided that the intersection of the two curves is not in the region of practical interest. 


\section{CASCADE SYSTEMS WITH GENERAL OUTPUT BACKLASH}

Cascade systems consisting of a linear dynamic system followed by a nonlinear static system are modeled by the so-called Wiener model and many identification methods have been proposed for this type of systems, eg [14-27].

In many real control systems, the backlash appears in a cascade connection with linear dynamic systems. One of the simplest cases is the cascade system where a linear dynamic system is followed by a general backlash as shown in Fig. 2. In this case, linear dynamic and nonlinear dynamic systems are cascaded; hence, an appropriate mathematical model is much more complicated than the Wiener model.

The linear dynamic system can be described by the difference equation as

$$
x(t)=\sum_{i=1}^{n a} a_{i} u(t-i)-\sum_{j=1}^{n b} b_{j} x(t-j)
$$

( $n a$ and $n b$ are assumed to be known) where $u(t)$ and $x(t)$ are the inputs and outputs, respectively.

Assume the general backlash is described by the difference equation (15). The input/output description of this cascade system can be constructed by connecting (16) and (15). However, a direct substitution of (16) into (15) leads to a quite complex expression, therefore it is appropriate to apply the so-called key term separation principle $[24,25]$. It means that (16) will be half-substituted, ie only for $x(t)$ in the first term of (15). Moreover, we can assume that $m_{L 1}=1$ (it is always possible in this connection of two subsystems), hence the input/output equation for the cascade system with general output backlash will be

$$
\begin{aligned}
& y(t)=\sum_{i=1}^{n a} a_{i} u(t-i) f_{1}(t)-\sum_{j=1}^{n b} b_{j} x(t-j) f_{1}(t)+ \\
& c_{L} f_{1}(t)+\sum_{i=2}^{n} m_{L i} \xi_{1}^{i}(t) f_{1}(t)+m_{R 1} x(t) f_{2}(t)-m_{R 1} c_{R} f_{2}(t)+ \\
& \sum_{i=2}^{n} m_{R i} \xi_{2}^{i}(t) f_{2}(t)+y(t-1)\left[1-f_{1}(t)\right]\left[1-f_{2}(t)\right]
\end{aligned}
$$

where the parameters of both the linear system and the general backlash are separated. Now the equation is quasilinear as the variables $\xi_{1}(t), \xi_{2}(t), f_{1}(t)$ and $f_{2}(t)$ depend on the backlash parameters and the internal variable $\mathrm{x}(\mathrm{t})$ depends on the linear system parameters.

We can define the following data vector

$$
\begin{aligned}
\phi(t)= & {\left[u(t-1) f_{1}(t), \ldots, u(t-n a) f_{1}(t),-x(t-1) f_{1}(t), \ldots,\right.} \\
- & x(t-n b) f_{1}(t), f_{1}(t), \xi_{1}^{2}(t) f_{1}(t), \ldots, \xi_{1}^{n}(t) f_{1}(t), ; \\
& \left.x(t) f_{2}(t),-f_{2}(t), \xi_{2}^{2}(t) f_{2}(t), \ldots, \xi_{2}^{n}(t) f_{2}(t)\right]^{\top}
\end{aligned}
$$

and the corresponding vector of parameters

$$
\begin{array}{r}
\theta=\left[a_{1}, \ldots, a_{n a}, b_{1}, \ldots, b_{n b}, c_{1}, m_{L 2}, \ldots, m_{L n}, m_{R 1},\right. \\
\left.c_{2}, m_{R 2}, \ldots, m_{R n}\right]^{\top}
\end{array}
$$

where

$$
m_{L 1}=1, \quad c_{L}=c_{1}, \quad c_{R}=c_{2} / m_{R 1}
$$

and the mathematical model for a cascade system with general output backlash can be written in the concise form

$$
y(t)-y(t-1)\left[1-f_{1}(t)\right]\left[1-f_{2}(t)\right]=\phi^{\top}(t) \theta+e(t)
$$

where $e(t)$ is a zero mean white noise.

As the variables $\xi_{1}(t), \xi_{2}(t), f_{1}(t), f_{2}(t)$ and the internal variable $x(t)$ in (18) are unmeasurable they must be estimated. Therefore, an iterative algorithm has to be considered for the parameter estimation. Assign the estimate of internal variable $x(t)$ in the $s$-th step as

$$
{ }^{s} x(t)=\sum_{i=1}^{n a}{ }^{s} a_{i} u(t-i)-\sum_{j=1}^{n b}{ }^{s} b_{j}{ }^{s} x(t-j)
$$

and the estimated variables $\xi_{1}(t), \xi_{2}(t), f_{1}(t)$ and $f_{2}(t)$ in the $s$-th step as

$$
\begin{aligned}
& { }^{s} \xi_{1}(t)={ }^{s} x(t)+{ }^{s} c_{L}, \\
& { }^{s} \xi_{2}(t)={ }^{s} x(t)-{ }^{s} c_{R}, \\
& { }^{s} f_{1}(t)=h\left[\sum_{i=1}^{n}{ }^{s} m_{L i}{ }^{s} \xi_{1}^{i}(t)-y(t-1)\right], \\
& { }^{s} f_{2}(t)=\left[y(t-1)-\sum_{i=1}^{n}{ }^{s} m_{R i}{ }^{s} \xi_{2}^{i}(t)\right] .
\end{aligned}
$$

Then the following error

$$
{ }^{s+1} \varepsilon(t)=y(t)-y(t-1)\left[1-{ }^{s} f_{1}(t)\right]\left[1-{ }^{s} f_{2}(t)\right]-{ }^{s} \varphi^{\top}(t){ }^{s+1} \theta
$$

will be minimized in the estimation procedure, where ${ }^{s} \varphi(t)$ is the data vector with the corresponding estimates of variables $x(t), \xi_{1}(t), \xi_{2}(t), f_{1}(t)$ and $f_{2}(t)$ according to $(22)-(26)$ and ${ }^{s+1} \theta$ is the $(s+1)$-st estimate of the parameter vector.

The steps of the algorithm for the cascade system identification are

a) Minimizing the least squares criterion based on (27), ie

$$
{ }^{s+1} J=\frac{1}{N} \sum_{t=1}^{N}{ }^{s+1} \varepsilon^{2}(t)
$$

where $N$ is the number of measured input/output samples, the estimates of parameters ${ }^{s+1} \theta$ are computed using ${ }^{s} \varphi(t)$ with the $s$-th estimates of variables ${ }^{s} x(t)$, ${ }^{s} \xi_{1}(t),{ }^{s} \xi_{2}(t),{ }^{s} f_{1}(t)$ and ${ }^{s} f_{2}(t)$. 


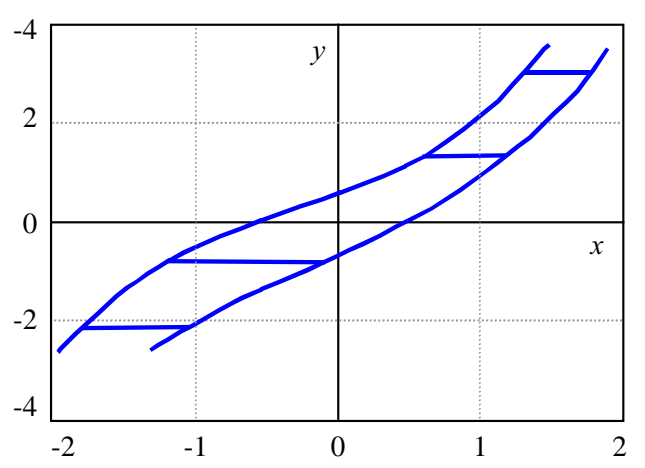

Fig. 4. Example 1 - general output backlash

b) Using (22)-(26), the estimates of ${ }^{s+1} \varphi(t)$ are evaluated by means of the recent estimates of corresponding parameters.

c) If the estimation criterion is met the procedure ends, else it continues by repeating steps a) and b).

In the first iteration, only the parameters of linear dynamic system are estimated. However, nonzero initial values of the general backlash parameters $m_{R 1}, c_{L}$ and $c_{R}$ have to be considered for evaluation of ${ }^{1} \varphi(t)$ to begin the iterative algorithm.

\section{SANDWICH SYSTEMS WITH GENERAL BACKLASH}

The so-called sandwich system consists of a linear dynamic system followed by a nonlinear system and this is followed by another linear dynamic system. If the nonlinear system is static, we speak about the WienerHammerstein system. The identification of these systems is more complicated than that of Wiener systems and fewer methods can be applied to deal with them, eg [2832]. A more complex case of sandwich system is shown in Fig. 3 where a linear dynamic system is followed by a general backlash, ie, a dynamic nonlinearity, and this is followed by another linear dynamic system.

Let the input linear system be described by (16) and the general backlash by (15), leading to (17). The output linear dynamic system can be described by the following difference equation ( $n r$ and $n s$ are assumed to be known)

$$
w(t)=\sum_{i=1}^{n r} r_{i} y(t-i)-\sum_{j=1}^{n s} s_{j} w(t-j) .
$$

Then the output equation of the sandwich system with a general backlash can be constructed in the similar way as that of cascade system in the previous Section. It means that the decomposition technique with halfsubstitution for the key term only will be applied in (29), where $y(t-1)$ will be chosen as the key term. We can again choose the value of one parameter in (29), ie we assume $r_{1}=1$. Now the sandwich system output equation will be

$$
\begin{gathered}
w(t)=\sum_{i=1}^{n a} a_{i} u(t-i-1) f_{1}(t-1)-\sum_{j=1}^{n b} b_{j} x(t-j-1) f_{1}(t-1) \\
+c_{L} f_{1}(t-1)+\sum_{i=2}^{n} m_{L i} \xi_{1}^{i}(t-1) f_{1}(t-1)+ \\
m_{R 1} x(t-1) f_{2}(t-1)-m_{R 1} c_{R} f_{2}(t-1)+ \\
\sum_{i=2}^{n} m_{R i} \xi_{2}^{i}(t-1) f_{2}(t-1)+y(t-2)\left[1-f_{1}(t-1)\right]\left[1-f_{2}(t-1)\right] \\
+\sum_{i=2}^{n r} r_{i} y(t-i)-\sum_{j=1}^{n s} s_{j} w(t-j)
\end{gathered}
$$

where the parameters of input and output linear systems and those of general backlash are separated and the equation is quasi-linear as the variables $\xi_{1} 1(t), \xi_{2}(t), f_{1}(t)$ and $f_{2}(t)$ and the internal variables $x(t)$ and $y(t)$ depend on the corresponding parameters. Defining the vector of parameters

$$
\begin{aligned}
\Theta & =\left[a_{1}, \ldots, a_{n a}, b_{1}, \ldots, b_{n b}, c_{1}, m_{L 2}, \ldots, m_{L n}\right. \\
& \left.m_{R 1}, c_{2}, m_{R 2}, \ldots, m_{R n}, r_{2}, \ldots, r_{n r}, s_{1}, \ldots, s_{n s}\right]^{\top}
\end{aligned}
$$

where

$$
m_{L 1}=1, \quad r_{1}=1, \quad c_{L}=c_{1}, \quad c_{R}=c_{2} / m_{R 1},
$$

and the vector of data

$$
\begin{gathered}
\Phi(t)=\left[u(t-2) f_{1}(t-1), \ldots, u(t-n a-1) f_{1}(t-1),\right. \\
-x(t-2) f_{1}(t-1), \ldots,-x(t-n b-1) f_{1}(t-1), \\
f_{1}(t-1), \xi_{1}^{2}(t-1) f_{1}(t-1), \ldots, \xi_{1}^{n}(t-1) f_{1}(t-1), \\
x(t-1) f_{2}(t-1),-f_{2}(t-1), \xi_{2}^{2}(t-1) f_{2}(t-1), \ldots, \\
\xi_{2}^{n}(t-1) f_{2}(t-1), y(t-2), \ldots, y(t-n r), \\
-w(t-1), \ldots,-w(t-n s)]^{\top}
\end{gathered}
$$

the mathematical model for the sandwich system with general backlash can be written in the vector form as

$w(t)-y(t-2)\left[1-f_{1}(t-1)\right]\left[1-f_{2}(t-1)\right]=\Phi^{\top}(t) \Theta+e(t)$,

where $e(t)$ is a zero mean white noise.

Again the variables $\xi_{1}(t), \xi_{2}(t), f_{1}(t), f_{2}(t)$ and the internal variables $x(t), y(t)$ are unmeasurable and must be estimated similarly as in the previous Section. Assume the estimates of $x(t)$ in the $s$-th step are given by (22) and the estimated variables $\xi_{1}(t), \xi_{2}(t), f_{1}(t)$ and $f_{2}(t)$ in the $s$-th step are given by $(23)-(26)$. The estimate of 


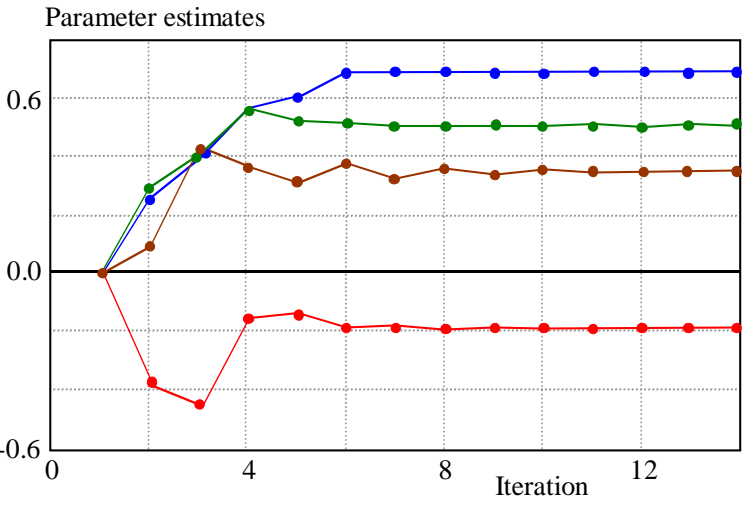

Fig. 5. Example 1 - parameter estimates - input linear system

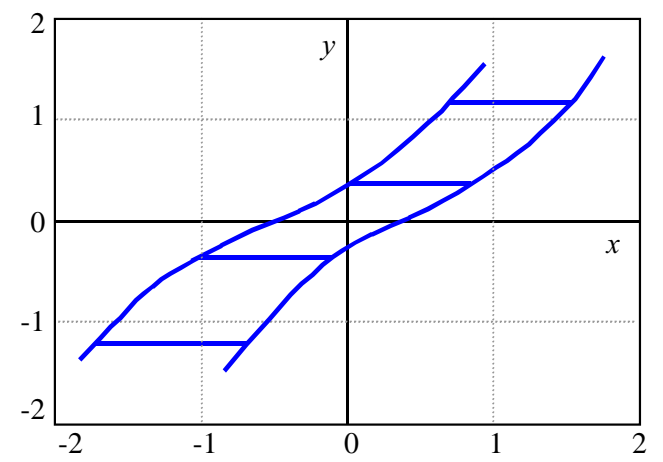

Fig. 7. Example 1 - parameter estimates - general backlash

internal variable $y(t)$ in the $s$-th step can be written as follows

$$
\begin{gathered}
{ }^{s} y(t)=\sum_{i=1}^{n a}{ }^{s} a_{i} u(t-i)^{s} f_{1}(t)-\sum_{j=1}^{n b}{ }^{s} b_{j}{ }^{s} x(t-j)^{s} f_{1}(t)+ \\
{ }^{s} c_{L}{ }^{s} f_{1}(t)+\sum_{i=2}^{n}{ }^{s} m_{L i}{ }^{s} \xi_{1}^{i}(t)^{s} f_{1}(t)+{ }^{s} m_{R 1}{ }^{s} x(t)^{s} f_{2}(t)- \\
{ }^{s} m_{R 1}{ }^{s} c_{R}{ }^{s} f_{2}(t)+\sum_{i=2}^{n}{ }^{s} m_{R i}{ }^{s} \xi_{2}^{i}(t)^{s} f_{2}(t)+ \\
{ }^{s} y(t-1)\left[1-{ }^{s} f_{1}(t)\right]\left[1-{ }^{s} f_{2}(t)\right] .
\end{gathered}
$$

Then the following error

$$
\begin{array}{r}
{ }^{s+1} \varepsilon(t)=w(t)-{ }^{s} y(t-2)\left[1-{ }^{s} f_{1}(t-1)\right]\left[1-{ }^{s} f_{2}(t-1)\right]- \\
{ }^{s} \Phi^{\top}(t){ }^{s+1} \Theta
\end{array}
$$

will be minimized in the estimation procedure, where ${ }^{s} \Phi(t)$ is the data vector with the corresponding estimates of variables $x(t), \xi_{1}(t), \xi_{2}(t), f_{1}(t), f_{2}(t)$ and $y(t)$ according to $(22)-(26)$ and $(35)$ and ${ }^{s+1} \Theta$ is the $(s+1)$-st estimate of the parameter vector.

The steps of the algorithm for the sandwich system identification are:

a) Minimizing the least squares criterion based on (36) the estimates of parameters ${ }^{s+1} \Theta$ are computed using

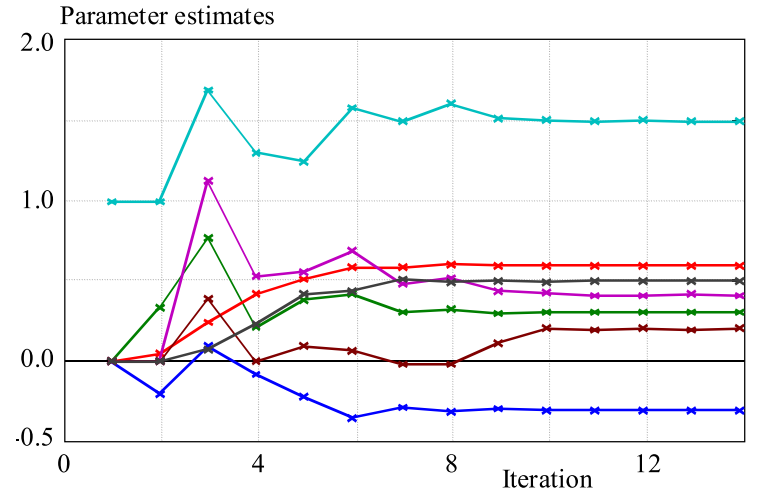

Fig. 6. Example 1 - parameter estimates - general backlash

${ }^{s} \Phi(t)$ with the $s$-th estimates of variables ${ }^{s} x(t),{ }^{s} \xi_{1}(t)$, ${ }^{s} \xi_{2}(t),{ }^{s} f_{1}(t),{ }^{s} f_{2}(t)$ and ${ }^{s} y(t)$.

b) Using (22)-(26) and (35) the estimates of ${ }^{s+1} \Phi(t)$ are evaluated by means of the recent estimates of corresponding parameters.

c) If the estimation criterion is met the procedure ends, else it continues by repeating steps a) and b).

In the first iteration, only the parameters of the input linear dynamic system are estimated. Again, nonzero initial values of the general backlash parameters $m_{R 1}, c_{L}$ and $c_{R}$ have to be considered for evaluation of ${ }^{1} \Phi(t)$ to begin the iterative algorithm.

\section{SIMULATION STUDIES}

The algorithms for the identification of cascade and sandwich systems with backlash were implemented and tested in MATLAB. The feasibility of the proposed methods is illustrated on the following examples.

ExAmple 1. The cascade system with the linear dynamic system given by the difference equation

$x(t)=0.7 u(t-1)+0.5 u(t-2)+0.2 x(t-1)-0.35 x(t-2)$

was followed by the general backlash (Fig. 4) with parameters $m_{L 2}=-0.3, m_{L 3}=0.3, c_{L}=0.6, m_{R 1}=1.5$, $m_{R 2}=0.4, m_{R 3}=0.2, c_{R}=0.5$. The identification was performed on the basis of 1500 samples of uniformly distributed random inputs with $|u(t)|<1.0$ and simulated outputs. Normally distributed random noise with zero mean and the signal to noise ratio (the square root of the ratio of output and noise variances) $\mathrm{SNR}=25$ was added to the outputs. The iterative estimation algorithm was applied with the initial values $m_{R 1}=1.0$ and $c_{L}=c_{R}=0.001$ for the first estimate of $\xi_{1}(t), \xi_{2}(t)$, $f_{1}(t)$ and $f_{2}(t)$. The process of linear system parameter estimation is shown in Fig. 5, while that of general backlash is shown in Fig. 6 (the top-down order of parameters is $\left.m_{R 1}, c_{L}, c_{R}, m_{R 2}, m_{L 3}, m_{R 3}, m_{L 2}\right)$. The estimates converge to the values of given parameters after 10 iterations. 


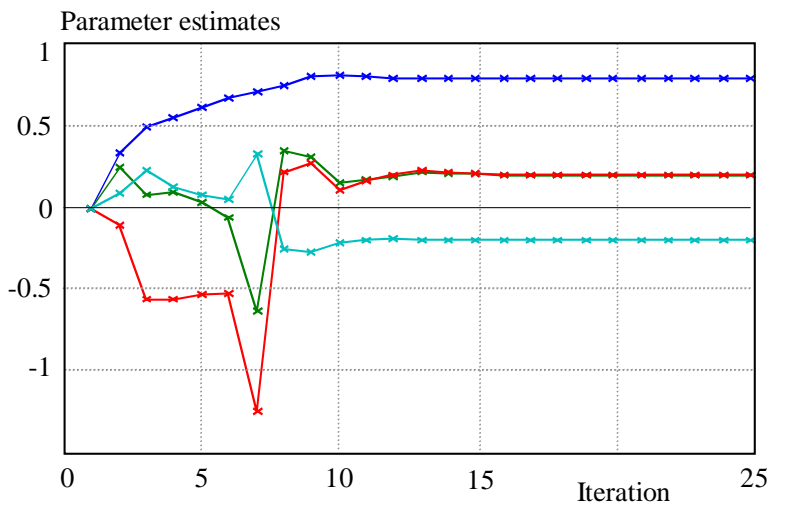

Fig. 8. Example 2 - parameter estimates - input linear system

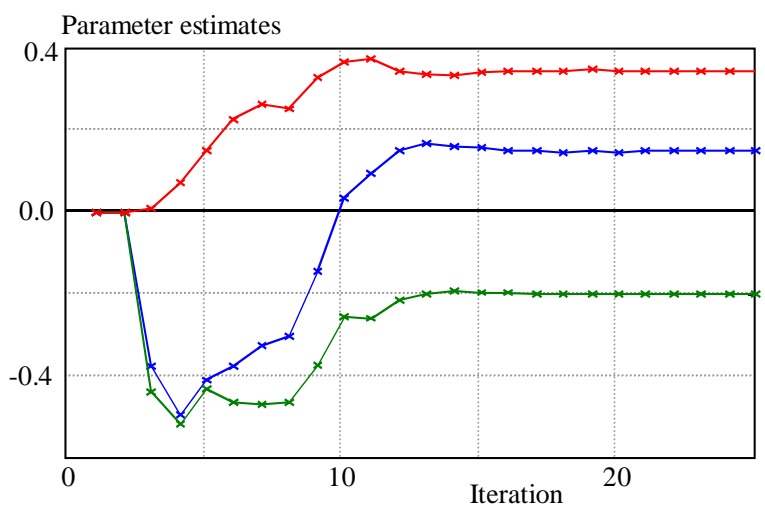

Fig. 10. Example 2 - parameter estimates - output linear system

EXAMPlE 2. The sandwich system with internal backlash was considered with the input linear dynamic system given by the difference equation

$x(t)=0.8 u(t-1)+0.2 u(t-2)-0.2 x(t-1)+0.2 x(t-2)$.

The general backlash (Fig. 7) with parameters $m_{L 2}=$ $0.1, m_{L 3}=0.3, c_{L}=0.4, m_{R 1}=0.75, m_{R 2}=0.2$, $m_{R 3}=0.6, c_{R}=0.5$ was followed by the output linear system given by the difference equation

$w(t)=y(t-1)+0.15 y(t-2)+0.2 w(t-1)-0.35 w(t-2)$.

The identification was performed on the basis of 1500 samples of uniformly distributed random inputs with $|u(t)|<1.0$ and simulated outputs. Normally distributed random noise with zero mean and $\mathrm{SNR}=100$ was added to the outputs. The iterative estimation algorithm was applied with the initial values $m_{R}=1.25$ and $c_{L}=c_{R}=$ 0.1 for the first estimates of $\xi_{1}(t), \xi_{2}(t), f_{1}(t)$ and $f_{2}(t)$. The process of parameter estimation is shown in Fig. 8 for the parameters of the input linear system, in Fig. 9 for the general backlash parameters (the top-down order of parameters is $\left.m_{R 1}, m_{R 3}, c_{R}, c_{L}, m_{L 3}, m_{R 2}, m_{L 2}\right)$ and in Fig. 10 for the parameters of the output linear system. The estimates converge to the values of given parameters after about 15 iterations.

Although a general convergence proof for parameter estimation algorithms with internal variable estimation is

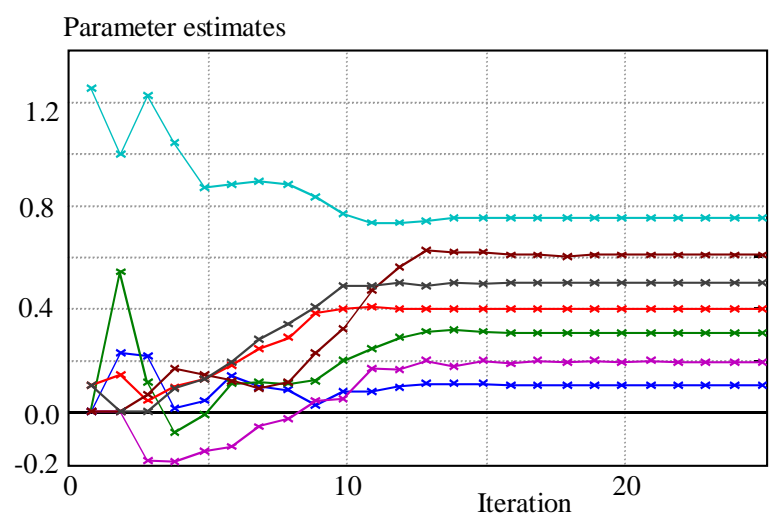

Fig. 9. Example 2 - parameter estimates - general backlash

not available [24,25], the above examples show that the convergence rate is relatively high despite the additive output noise.

\section{CONCLUSIONS}

In this paper, a recently proposed analytic form of general backlash characteristic description was used in mathematical models of cascade and sandwich systems including this type of dynamic nonlinearity. Iterative algorithms were proposed for both cases enabling simultaneous estimation of the general backlash parameters and the parameters of the cascaded linear dynamic systems on the basis of input/output data. The feasibility of both identification methods was shown in simulation studies.

The identification of systems with unknown backlash is of major relevance to many control applications dealing with mechanical, hydraulic, pneumatic, magnetic, and piezoelectric systems. Introduction of the general backlash leads to more precise descriptions and significantly contributes to better results in control of the cascade system with output general backlash and the sandwich system with internal general backlash. Finally note that the proposed forms of cascade and sandwich models can be used in recursive identification algorithms, too.

\section{Acknowledgement}

The author gratefully acknowledges financial support from the Slovak Scientific Grant Agency (VEGA).

\section{REFERENCES}

[1] NORDIN, M.-GUTMAN, P. O.: Controlling Mechanical Systems with Backlash - a Survey, Automatica 38 (2002), 1633-1649.

[2] KALAŠ, V.-JURIŠICA, L.—ŽALMAN, M.-ALMÁSSY, S.SIVIČEK, P.-VARGA, A. KALAŠ,-D. : Nonlinear and Numerical Servosystems, Alfa/SNTL, Bratislava, 1985. (in Slovak)

[3] CERone, V.-Regruto, D.: Bounding the Parameters of Linear Systems with Input Backlash, IEEE Trans. Automatic Control 52 (2007), 531-536. 
[4] DONG, R.-TAN, Y.-CHEN, H. : Recursive Identification for Dynamic Systems with Backlash, Asian Journal of Control 12 (2010), 26-38.

[5] GIRI, F.-ROCHDI, Y.-CHAOUI, F. Z.-BROURI, A. : Identification of Hammerstein Systems in Presence of Hysteresis-Backlash and Hysteresis-Relay Nonlinearities, Automatica 44 (2008), 767-775.

[6] HUANG, X.-WANG, J.: Identification of Ground Vehicle Steering System Backlash, Transactions of the ASME Journal of Dynamic Systems, Measurement, and Control 135 (2013), art. no. 011014 (8 pages).

[7] VÖRÖS, J. : Modeling and Identification of Systems with Backlash, Automatica 46 (2010), 369-374.

[8] VÖRÖS, J.: Identification of Cascade Systems with Backlash, International Journal of Control 83 (2010), 1117-1124.

[9] VÖRÖS, J.: Identification of Nonlinear Cascade Systems with Time-Varying Backlash, J. Electrical Engineering 62 (2011), $87-92$.

[10] GIRI, F.-ROCHDI, Y.-BROURI, A.-CHAOUI, F. Z. : Parameter Identification of Hammerstein Systems Containing Backlash Operators with Arbitrary-Shape Parametric Borders, Automatica 47 (2011), 1827-1833.

[11] ROCHDI, Y.-GIRI, F.-GNING, J. B.-CHAOUI, F. Z. Identification of Block-Oriented Systems in the Presence of Nonparametric Input Nonlinearities of Switch and Backlash Types, Automatica 46 (2010), 785-958.

[12] VÖRÖS, J. : Parametric Identification of Systems with General Backlash, Informatica 23 (2012), 283-298.

[13] VÖRÖS, J. : Recursive Identification of Nonlinear Cascade Systems With Time-Varying General Input Backlash, ASME Journal of Dynamic Systems, Measurement, and Control 135 (2013), art. no. 014504 (5 pages).

[14] BAI, E. W.-REYLAND, J. : Towards Identification of Wiener Systems with the Least Amount of a Priori Information: IIR Cases, Automatica 45 (2009), 956-964.

[15] CHEN, H. F.: Recursive Identification for Wiener Model with Discontinuous Piece-Wise Linear Function, IEEE Trans. Automatic Control 51 (2006), 390-400.

[16] CHEN, J.-ZHANG, Y.-DING, F.: Gradient based Iterative Algorithm for Wiener Systems with Piece-Wise Nonlinearities using Analytic Parameterization Methods, Computers and Applied Chemistry 28 (2011), 855-857.

[17] HU, X. L.-CHEN, H. F. : Recursive Identification for Wiener Systems using Gaussian Inputs, Asian Journal of Control 10 (2008), 341-350.

[18] HWANG, S-H.-HSIEH, C-Y.-CHEN, H-T.-HUANG, Y-C. : Use of Discrete Laguerre Expansions for Noniterative Identification of Nonlinear Wiener Models, Industrial \& Engineering Chemistry Research 50 (2011), 1427-1438.

[19] JANCZAK, A. : Instrumental Variables Approach to Identification of a Class of MIMO Wiener Systems, Nonlinear Dynamics 48 (2007), 275-284.

[20] KAZLAUSKAS, K.-PUPEIKIS, R. : On Intelligent Extraction of an Internal Signal in a Wiener System Consisting of a Linear Block Followed by Hard-Nonlinearity, Informatica, 24 (2013), $35-58$.
[21] MZYK, G.: A Censored Sample Mean Approach to Nonparametric Identification of Nonlinearities in Wiener Systems, IEEE Trans. Circuits and Systems II: Analog and Digital Signal Processing 54 (2007), 897-901.

22] PAWLAK, M.-HASIEWICZ, Z.-WACHEL, P.: On Nonparametric Identification of Wiener Systems, IEEE Trans. Signal Processing 55 (2007), 482-492.

23] PUPEIKIS, R. : On the Identification of Wiener Systems Having Saturation-Like Functions with Positive Slopes, Informatica 16 (2005), 131-144.

[24] VÖRÖS, J.: Modeling and Identification of Wiener Systems with Two-Segment Nonlinearities, IEEE Trans. Control Systems Technology 11 (2003), 253-257.

25] VÖRÖS, J.: Parameter Identification of Wiener Systems with Multisegment Piecewise-Linear Nonlinearities, Systems and Control Letters 56 (2007), 99-105.

26] WANG, D.-DING, F.: Least Squares based and Gradient Based Iterative Identification for Wiener Nonlinear Systems, Signal Processing 91 (2011), 1182-1189.

27] ZHOU, L.-LI, X.-PAN, F. : Gradient based Iterative Parameter Identification for Wiener Nonlinear Systems, Applied Mathematical Modelling 37 (2013), 8203-8209.

[28] BAI, E. W.-CAI, Z.-DUDLEY-JAVOROSK, S.-SHIELDS, R. K. : Identification of a Modified Wiener-Hammerstein System and its Application in Electrically Stimulated Paralyzed Skeletal Muscle Modeling, Automatica 45 (2009), 736-743.

[29] SJBERG, J.-SCHOUKENS, J.: Initializing Wiener-Hammerstein Models based on Partitioning of the Best Linear Approximation, Automatica 48 (2012), 353-359.

[30] TAN, Y. H-DONG, R. L.-LI, R. Y. : Recursive Identification of Sandwich Systems with Dead Zone and Application, IEEE Trans. Control Systems Technology 17 (2009), 945-951.

[31] VÖRÖS, J.: An Iterative Method for Wiener-Hammerstein Systems Parameter Identification, J. Electrical Engineering $\mathbf{5 8}$ (2007), 114-117.

32] XIE, Y.-TAN, Y.-DONG, R. L.: Identification of Sandwich Systems with Dead Zone using Combinational Input Signals, Transactions of the Institute of Measurement and Control 33 (2011), 957-970

Received 16 September 2013

Jozef Vörös (Ing, PhD) was born in Hurbanovo on July 9 , 1949. He graduated in automatic control from the Faculty of Electrical Engineering of Slovak Technical University, Bratislava in 1974 and received his $\mathrm{PhD}$ degree in control theory from the Institute of Technical Cybernetics of the Slovak Academy of Sciences, Bratislava in 1983. Since 1992 he has been with the Faculty of Electrical Engineering and Information Technology at the Slovak Technical University in Bratislava where he is acting as a senior research scientist in the Institute of Robotics and Cybernetics. His research interests include the analysis and identification of nonlinear systems. He is also interested in the area of mobile robot path planning using quadtree and octree representations. 